\title{
AUTOMATED DATA COLLECTION FOR INFRASTRUCTURE PROJECT CONTROL
}

\author{
Ronie Navon \\ Associate Professor \\ Head, Construction Management \& Economics - National Building Research Institute (NBRI); \\ and Head, Construction Automation Laboratory - Faculty of Civil \& Environmental Engineering, \\ Technion City, 32000 Haifa, Israel. \\ ronie@technion.ac.il.
}

\begin{abstract}
Infrastructure construction is typically of large financial scale and is less structured than building construction projects. Hence, real-time control of on-site infrastructure construction, based on high quality data, is essential to identify discrepancies between actual and planned performances. Additionally, real-time control enables timely corrective measures to be taken when needed to reduce the damages caused by the discrepancies. We have developed models that use automated data collection technologies GPS mounted on the earthmoving equipment - to measure the location of the equipment as function of time. These models are capable of measuring productivity and progress quite accurately. The oral presentation will include a wider description of work in the area of automated data collection applications for purposes different than ours.
\end{abstract}

\section{INTRODUCTION}

Real-time information is necessary to efficiently monitor performance indicators. The absence of such information limits construction managers' ability to monitor performance indicators, which increases the uncertainty inherent in project activities. It is not surprising, therefore, that construction projects are very often late, exceed their budgets and that productivity has remained static, even in developed countries, over the last 20 years (Allmon et al. 2000; Josephson and Hammarlund 1999).

Many construction managers perform only generic and infrequent control because traditional control methods, which are based on manual data collection, are slow and inaccurate. Because current data collection methods are time consuming and expensive, many construction companies do not collect extensive data and even less so in real-time. Even recent developments in automated data collection (Ciesielski 2000) have not alleviated this situation (Saidi et al. 2003).

\section{PREVIOUS RESEARCH IN AUTOMATED ROAD CONSTRUCTION CONTROL}

In recent years we have been working on various stages of developing a model for automated control of road construction. The first model assumed that the plan of the road is divided into work sections (WS) - these are areas where work on the road is performed at a given time interval, such as a day (Navon et al. 2004; Navon and Shpatnitsky 2005; Navon and Shpatnitsky 2006). The research introduced the concept of the work envelop (WE), which is an extension of the WS where a piece of equipment working on the WS can be located while working on it. Thus, a WE is a geometrical extension of the corresponding WS.

A GPS measures the locations of the equipment to determine what work the equipment was engaged in at the time the location was measured. This is done by associating the locations to the WE and WS in two stages: I. The geometrical stage - all locations, which are located within one WE are associated to the corresponding WS. All other 
locations (the ones, which cannot be geometrically associated to more than one WE and those which are not located within any WE), are associated at the logical stage. The association at the latter stage is done with the aid of decision rules. Knowing the time equipment spent performing a given activity and the amount of work done, the model calculates the productivity performing each WS.

The model was implemented in a prototype system and tested on site. The location of the equipment as function of time was measured with two GPS receivers using Differential GPS (DGPS) technology for increased accuracy. A series of site experiments were conducted with different types of equipment, different activities and for three types of WE. During the experiment we also manually measured the actual productivity in order to compare the actual performance and that of the model. The results of this comparison yielded the following: (i) The accuracy of the model using a WE without overlap was between c. $+1 \%$ for Asphalt Spreading - a well structured activity, and $+11 \%$ for Spread and Grade Sub-Base. (ii) The accuracy using a WE with overlap was between c. $-2 \%$ for Asphalt Spreading to $+4 \%$ for Spread and Grade Fill.

These results showed that the performance of road construction can be measured automatically using equipment-location measured with GPS. The difficulty with the site experiments was that the actual work did not progress according to the pattern of the predefined WS from the plans. In other words, the work sequence was more chaotic, especially in the case of the less structured activities. This difficulty was overcome, at the end of each working day, by manually defining the WE relating to the work performed during the given day.

\section{CURRENT RESEARCH IN AUTOMATED ROAD CONSTRUCTION CONTROL}

The need for manually defining the work performed at the end of each day lead us to thoughts about a new concept for WE and WS. Instead of associating locations to activities in a two-stage association procedure by predetermining WS and correspondingly WE, it was decided that the algorithm must determine the WE dynamically during its operation, according to the measured locations - we called it the dynamic work envelope (DWE) approach. Various existing algorithms were considered for the DWE. The first is called Minimum Convex Polygon. This algorithm determines the area of the smallest convex polygon which encompasses all the measured locations. The advantage of this algorithm is its simplicity. The problem with it, on the other hand, is that the calculated area includes the entire area encompassing all the measured locations, which means that even areas where work was not performed are included. The second algorithm is called Kernel Density Estimation algorithm, which is more sophisticated - it is based on statistical principles and hence is more accurate. This algorithm is capable of identifying and subtracting areas where there is no concentration of locations and is also capable of ignoring incidental readings.

An initial field test of the two algorithms was conducted. The results of this test indicate that these algorithms are capable of determining the area represented by the measured locations; hence they are potentially suitable to serve as part of the DWE algorithm. As for their accuracy, the areas calculated by the two algorithms were compared to a manual calculation based on surveying the area professionally. The deviations were $+166 \%$ and + $52 \%$ correspondingly, which deemed these algorithms as unsuitable for productivity control purposes.

We are currently in advanced stages of developing a different DWE model (Navon 2007). The new model was checked with the data from the initial field test and gave better results. The new model will have to be fully implemented in a computer program and tested in comprehensive field tests. The new data collected in the field tests will have to be processed and analyzed to evaluate the DWE model in order to draw conclusions pertaining to: (1) key parameters affecting the automated measurement, (2) expected accuracies of the automated measurement, and (3) the feasibility, or suitability, of GIS as the operating environment.

\section{CONCLUSIONS}

Automated data collection is essential for efficient real-time control of infrastructure control. The first model that we developed yielded good results in the field tests, but was not fully automatic. The 
second model has a much higher degree of automation and the initial results from the field tests are encouraging.

Both models described above are capable of measuring productivity and progress in projects that can be represented in $2 \mathrm{D}$, such as roads. We are planning to launch a basic research for doing the same in earthmoving projects involving cut and fill operations. Controlling these projects require 3D automated data collection.

\section{ACKNOWLEDGMENT}

This research was supported by THE ISRAEL SCIENCE FOUNDATION (grant No. 444/05). The support is gratefully acknowledged. I would like to thank my colleagues Professor Y. Doytsher, Eng. S. Khoury and Eng. Y. Shpatnitsky who have contributed so much to the research in the area of automated infrastructure control.

\section{REFERENCES}

[1] Allmon, E., Haas, C. T., Borcherding, J. D., and Goodrum, P. M. (2000). "U.S. Construction Labor Productivity Trends, 1970-1998." Journal of Construction Engineering and Management, ASCE, 126(2), 97-104.

[2] Ciesielski, C. A. (2000). "ADCIC - Automatic Data Collection in Construction." Haifa, Israel.
[3] Josephson, P. E., and Hammarlund, Y. (1999). "The causes and costs of defects in construction: A study of seven building projects." Automation in Construction, 8, 681687.

[4] Navon, R. (2007). "Research in Automated Measurement of Project Performance Indicators." Automation in Construction, 16(2), 176-188.

[5] Navon, R., Goldschmidt, E., and Shpatnitsky, Y. (2004). "A Concept Proving Prototype of Automated Earthmoving Control." Automation in Construction, 13(2), 225-239.

[6] Navon, R., and Shpatnitsky, Y. (2005). "Field Experiments in Automated Monitoring of Road Construction." Journal of Construction Engineering and Management, ASCE, 131(4), 487-493.

[7] Navon, R., and Shpatnitsky, Y. (2006). "A Model for Automated Monitoring of Road Construction." Construction Management and Economics, 23(9), 941-951.

[8] Saidi, K. S., Lytle, A. M., and Stone, W. C. (2003). "Report of the NIST Workshop on Data Exchange Standards at the Construction Job Site." ISARC 2003 the Future Site, Eindhoven, The Netherlands, 617-622. 